\title{
An Introduction to the Spectral Analysis of the QGP
}

\author{
P. P. Bhaduri ${ }^{1}$, P. Hegde ${ }^{2}$, H. Satz ${ }^{3}$ and P. Tribedy ${ }^{1}$ \\ ${ }^{1}$ VECC, 1/AF Bidhan Nagar, Kolkata-700 064, India. \\ ${ }^{2}$ Department of Physics and Astronomy, SUNY, Stony Brook, NY 11794-3800, \\ USA. \\ ${ }^{3}$ Fakultät für Physik, Universität Bielefeld, D-33501 Bielefeld, Germany.
}

Summary. This is an introduction to the study of the in-medium behavior of quarkonia and its application to the quark-gluon plasma search in high energy nuclear collisions.

\subsection{What are quarkonia?}

The bound states of a heavy quark and its anti-quark which are stable with respect to strong decay into open charm or bottom are collectively called quarkonia. We denote by $Q$ either of the heavy quarks, charm $(c)$ or bottom $(b)$; the corresponding bound states are known as charmonia or bottomonia, respectively.

Among the vector (spin-one) charmonium states, the lightest (ground state) is the famous $J / \psi$; the excited states are the $\chi_{c}$ and the $\psi^{\prime}$. For the bottom quark, the lightest quarkonium is the $\Upsilon$, while the excited states include the $\chi_{b}, \Upsilon^{\prime}, \chi_{b}^{\prime}$ and the $\Upsilon^{\prime \prime}$. The stability of the $c \bar{c} / b \bar{b}$ quarkonium states implies that their masses satisfy $M_{c \bar{c}}<2 M_{D}$ and $M_{b \bar{b}}<2 M_{B}$, where $D=c \bar{u}$ and $B=b \bar{u}$ are the corresponding "open" mesons. A specific characteristic of quarkonia is their small size. While the typical hadron radius is $\sim 1 \mathrm{fm}$, the radii of charmonia and bottomonia range from $0.1-0.3 \mathrm{fm}$, as we shall see.

Since $c$ and $b$ quarks are very heavy $\left(m_{Q} \ll \Lambda_{Q C D} \sim 200 \mathrm{MeV}\right)$, the binding of the $Q \bar{Q}$ system may be treated non-relativistically. The governing equation is the non-relativistic Schrödinger equation,

$$
-\frac{1}{m}\left\{\nabla^{2}(r)+V(r)\right\} \Psi_{i}(r)=\left(M_{i}-2 m\right) \Psi_{i}(r)
$$

where $\Psi(r)$ denotes the wavefunction of the system, $r$ the quark-antiquark separation, and $m$ the quark mass 1 . Since Eq. (1.1) is a nonrelativistic description of the binding, the total rest mass must be subtracted from the masses $M_{i}$ of the

\footnotetext{
${ }^{1}$ We work in the center-of-mass system, with a reduced mass $m / 2$, so that we have $-\nabla^{2} / m$ instead of the usual $-\nabla^{2} / 2 m$.
} 


\begin{tabular}{|c|c|c|c|c|c|c|c|c|}
\hline state & $J / \psi$ & $\chi_{c}$ & $\psi^{\prime}$ & $\Upsilon$ & $\chi_{b}$ & $\Upsilon^{\prime}$ & $\chi_{b}^{\prime}$ & $\Upsilon^{\prime \prime}$ \\
\hline \hline mass $(\mathrm{GeV})$ & 3.10 & 3.53 & 3.68 & 9.46 & 9.99 & 10.02 & 10.36 & 10.36 \\
\hline$\Delta E(\mathrm{GeV})$ & 0.64 & 0.20 & 0.05 & 1.10 & 0.67 & 0.54 & 0.31 & 0.20 \\
\hline radius $(\mathrm{fm})$ & 0.25 & 0.36 & 0.45 & 0.14 & 0.22 & 0.28 & 0.34 & 0.39 \\
\hline
\end{tabular}

Table 1.1. Masses, binding energies and radii of lowest $c \bar{c}$ and $b \bar{b}$ bound states $[2$.

bound states. Once we find the eigenvalues $M_{i}$ of the system, we can also define the "binding energy" $\Delta E$ of each quarkonium state, $\Delta E=2 M_{D, B}-M_{i}$.

Lattice and spectroscopic studies suggest for the potential $V(x)$ the form [1]

$$
V(r)=\sigma r-\frac{\alpha}{r},
$$

generally known as the "Cornell potential". It is spherically symmetric, and consists of two parts. The linearly rising part represents the confining force, given in terms of the string tension $\sigma$; lattice studies put its value at around $0.2(\mathrm{GeV})^{2}$. The second part is an effective Coulomb potential, including transverse string oscillations; string theory suggests $\alpha=\pi / 12$.

Having solved the Schrödinger equation, we may determine the bound-state radii through

$$
\left\langle r_{i}^{2}\right\rangle=\frac{\int d^{3} r r^{2}\left|\Psi_{i}(r)\right|^{2}}{\int d^{3} r\left|\Psi_{i}(r)\right|^{2}} .
$$

A fair estimate can already be obtained by means of a semi-classical formulation. The energy of the system is then given by

$$
E=\frac{p^{2}}{m}+V(r)
$$

and from the uncertainty relation we have $p r \simeq c$; the constant $c$ can be fixed by requiring the correct $J / \psi$ mass, giving $c \simeq 1.25$. Minimizing the energy determines the lowest bound state radius $r_{0}$,

$$
\sigma+\frac{\alpha}{r_{0}^{2}}=\frac{3}{m r_{0}^{3}}
$$

With $\sigma \simeq 0.2(\mathrm{GeV})^{2}$ and $\alpha \simeq \pi / 12$, together with $m_{c} \simeq 1.3 \mathrm{GeV}$, we obtain a $J / \psi$ size $\left(Q \bar{Q}\right.$ separation, i.e., twice the radius) of about $0.5 \mathrm{fm}$. For the $\alpha^{\prime}=0$ value, we have $r_{0} \sim(1 / m \sigma)^{1 / 3} \approx 0.3 \mathrm{fm}$; on the other hand, for $\sigma=0$, we get $r_{0} \sim(1 / m \alpha) \approx 0.6 \mathrm{fm}$. We thus see that a major contribution to the radius comes from the string tension. At $T=0$, the radius of the $J / \psi$ is thus to a considerable extent still determined by the confining part of the potential. We summarize some of the characteristics of the spin-averaged quarkonia in Table 1.1 [2].

Next, we turn to the question of the dissociation and decay of quarkonia. We have already noted that these mesons cannot decay via strong channels because their masses are smaller than the open thresholds. It is also known that quarkonia do not dissociate significantly in nuclear collisions; we shall discuss this in greater detail in section 1.3 . 
How then do quarkonia dissociate? Three mechanisms have been identified, corresponding to the behavior for $T=0,0<T<T_{c}$ and $T \geq T_{c}$, where $T_{c}$ is the critical temperature of deconfinement. We shall consider each of them in turn.

\subsubsection{String-breaking}

The potential in Eq. 1.2 is correct only in the limit $M_{Q} \rightarrow \infty$. If light quarks exist in the theory, then the string connecting the heavy quarks can break as soon as the overall energy in the system is greater than $2 M_{D}$ or $2 M_{B}$, depending on whether $Q=c$ or $b$ (Fig. 1.1). Light quark-antiquark pairs appear at the broken ends of the string, and new "heavy-light" $Q \bar{q}$ or $q \bar{Q}$ mesons are formed. This behavior of the quark potential has been observed in lattice studies with dynamical quarks, as we shall show below.
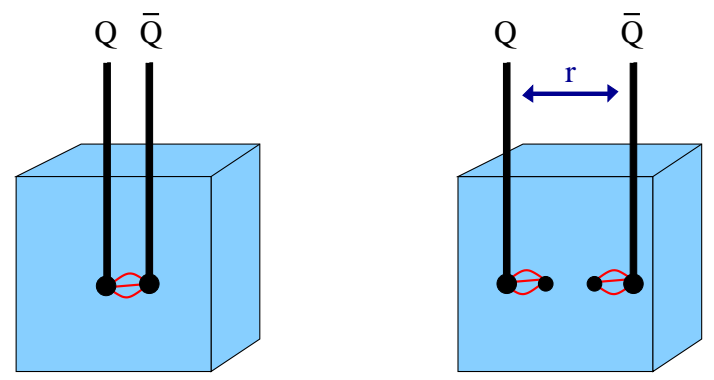

Fig. 1.1. Cartoon of string-breaking

We may estimate the string-breaking energy $F_{0}$. For the charm quark, $F_{0}=$ $2\left(M_{D}-m_{c}\right) \simeq 1.2 \mathrm{GeV}$, while for the bottom quark, $F_{0}=2\left(M_{B}-m_{b}\right) \simeq 1.2 \mathrm{GeV}$. From this, we deduce $r_{0}=(1.2 \mathrm{GeV}) / \sigma \simeq 1.5 \mathrm{fm}$. That this value is the same for both quark species leads us to conclude that the energy required for string breaking is a property of the vacuum itself, as a medium at $T=0$, containing virtual $q \bar{q}$ pairs which are brought on-shell by the field between the heavy quarks. The effect of string breaking on the Cornell potential is shown in Fig. 1.2

\subsubsection{Recombination}

In nuclear collisions not sufficiently energetic to create a quark-gluon plasma, there will nevertheless be abundant hadron production. These newly formed light hadrons can through a switch in bonding (recombination) turn a $Q \bar{Q}$ meson into two heavylight mesons. This mechanism is schematically depicted in Fig. 1.3 when two or more hadrons overlap, their quarks can recouple form new pairs.

If the temperature is increased, the hadron density also increases, and this in turn increases the recombination probability. As a consequence, the distance up to which the heavy quarks still bind also becomes shorter, and the potential will break 


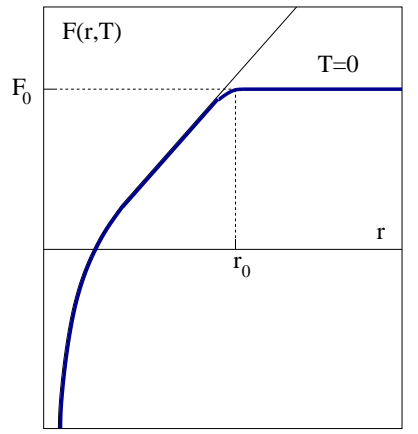

Fig. 1.2. String breaking energy as function of $Q \bar{Q}$ separation

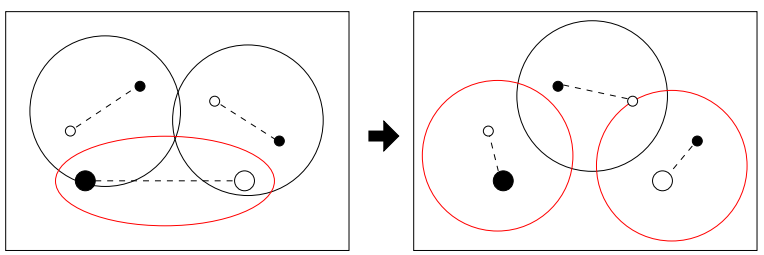

Fig. 1.3. A schematic view of recombination

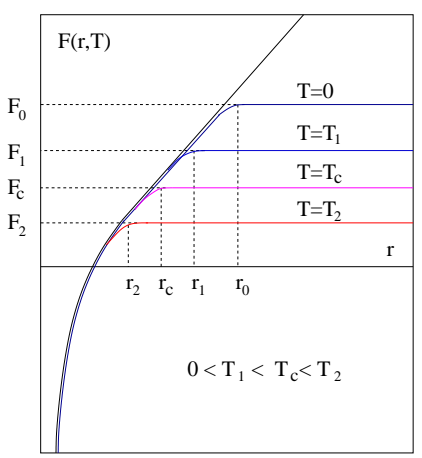

Fig. 1.4. Schematic dependence of the string-breaking radius with temperature

earlier (see Fig. 1.4 We thus have something like "effective screening", even though all color charges are still bound.

What happens as we get close to $T_{c}$ ? The density of produced hadrons will then increase strongly, and lattice studies show that in accord with our picture, both the free energy and the string-breaking radius $r_{T}$ decrease rapidly near $T_{c}$, as shown in Fig. 1.5.

\subsubsection{Color screening}

Above $T=T_{c}$, we have a medium of unbound color charges, and an entirely different mechanism takes over. At all temperatures $T$ above zero, quarks and gluons are 

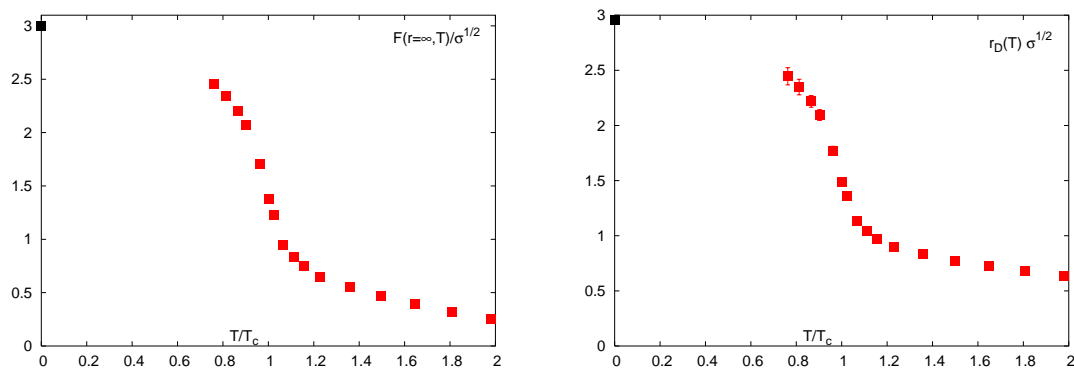

Fig. 1.5. Lattice results for free energy and screening radius as function of $T$.

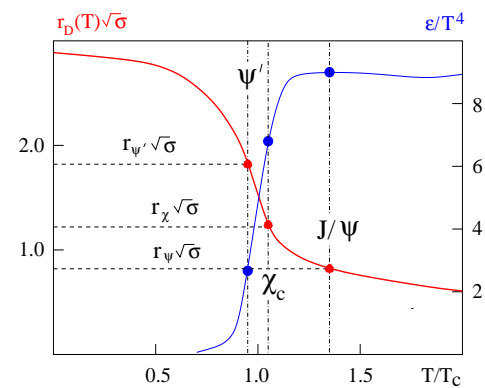

Fig. 1.6. Quarkonium dissociation as "thermometer" for the quark-gluon plasma.

screened, just as electric charges experience Debye screening in an electromagnetic plasma. This screening occurs with a characteristic radius, which we denote by $r_{D}$. It decreases with increasing temperature, as the medium increases in density. Deconfinement is expected to occur when this radius becomes comparable to the average hadron size of $1 \mathrm{fm}$. Then a given quark can no longer see its former partner in a hadron; instead, it sees many other quarks and antiquarks and therefore can move around freely, without encountering any confinement limit, since it is never 1 fm away from an antiquark.

We would like to use the behavior of the $J / \psi$ to probe if a quark-gluon plasma was formed in the collision [3. However, as we have seen, the $J / \psi$ and its heavier counterparts have smaller radii than the usual hadrons. Thus, charmonia and bottomonia may be expected to survive beyond the QGP phase transition up to some higher temperature, at which they will become dissociated. Thus, if we know their sizes as well as the behaviour of $r_{D}$ as a function of $T$, we can use their dissociation points to determine the temperature and the energy density $\epsilon$ of the QGP medium [4, as illustrated in Fig. 1.6. 


\subsection{Studying charmonium dissociation}

We now turn to the question of how to determine quantitatively the quarkonium dissociation points in a quark-gluon plasma. Two different approaches were used to address this problem.

- Solve the Schrödinger equation with a temperature-dependent potential $V(r, T)$, or

- calculate the quarkonium spectrum directly in finite temperature lattice QCD.

We shall look at each of these approaches in turn.

\subsubsection{Potential models for quarkonium dissociation}

\section{The Schwinger model [5]}

One generalizes the Cornell potential, eq. (1.2), to non-zero temperature in the form

$$
V(r, T)=\sigma r\left\{\frac{1-e^{-\mu r}}{\mu r}\right\}-\frac{\alpha}{r} e^{-\mu r} .
$$

The $T$-dependence of the above equation is in the "screening mass" $\mu(T)=1 / r_{D}(T)$. Eq. (1.6) gives the correct zero-temperature limit, eq. (1.2), for $\mu(T) \rightarrow 0$ as $T \rightarrow 0$.

To determine the dissociation points, one solves the Schrödinger equation and determines the bound-state energies $M_{i}(\mu)$. With increasing temperature, the bound state $i$ disappears at some $\mu=\mu_{i}$. One then uses the temperature dependence of the screening mass from lattice estimates, $\mu(T) \simeq 4 T$, to determine the $T_{i}$. The result of this model is

- the $\psi^{\prime}$ and $\chi_{c}$ become dissociated around $T \simeq T_{c}$,

- the $J / \psi$ survives up to about $T \simeq 1.2 T_{c}$.

In both cases, at the dissociation point the binding energy vanishes, while the binding radius diverges.

\section{Lattice potential models [6, 7, 8, 9, 10]}

Alternatively, one may use lattice results for the temperature dependence of the potential felt by a static quark-antiquark pair to determine the needed potential. The static $Q \bar{Q}$ studies start from the partition function $Z$, which is related to the free energy by $Z=\exp (-\beta F)$; this in turn gives the thermodynamic potentials

$$
\begin{gathered}
F=U-T S \\
S=-\left(\frac{\partial F}{\partial T}\right)_{V} \\
U(r, T)=F(r, T)-T\left(\frac{\partial F(r, T)}{\partial T}\right)_{V}
\end{gathered}
$$

Assuming that the internal energy $U(r, T)$ provides the temperature dependence of the heavy quark potential, we we use results from $N_{f}=2$ lattice QCD and solve the Schrödinger equation. The results obtained from such studies indicate that 

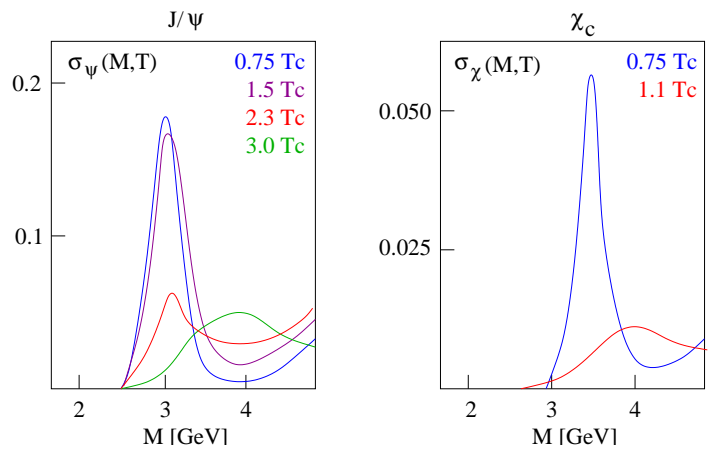

Fig. 1.7. Schematic view of lattice results for charmonium dissociation.

- the $\psi^{\prime}$ and $\chi_{c}$ are dissociated around a temperature $T \simeq 1.1 T_{c}$,

- $J / \psi$ survives up to a temperature $T \simeq 2 T_{c}$.

Comparing these results to the ones from the Schwinger model, we see that while there is agreement in the case of the higher excited states, lattice potential models predict a considerably higher dissociation temperature for the $J / \psi$. The reason for this is that the internal energy $U(r, T)$ leads to much stronger binding than the Schwinger model potential.

It should be noted here that there still is some ambiguity as to whether $U$ or $F$ is the correct potential to be used in the Schrödinger equation. Hence there exist approaches with potentials of the form $a U+(1-a) F$, with $0 \leq a \leq 1$. Such potentials tend to reduce binding and lower the dissociation temperature as $a$ is decreased.

\subsubsection{Lattice studies of charmonium survival}

The ideal way to resolve the above ambiguity would be to calculate the $c \bar{c}$ spectrum directly on the lattice, and this is indeed what lattice studies aim to do 11, 12, 13, 14, 15, 16. More specifically, they calculate the $c \bar{c}$ spectrum $\sigma(\omega, T)$ in the appropriate quantum channel, as a function of the temperature $T$ and the $c \bar{c}$ energy $\omega$. Boundstates show up as resonances in a plot of $\sigma$ versus $\omega$. By performing simulations at different temperatures, one can determine the temperature at which a particular peak disappears i.e. a bound-state dissolves. A schematic illustration is shown in Fig. 1.7. The results presently indicate that

- $\chi_{c}$ is dissociated for $T \geq 1.1 T_{c}$.

- $J / \psi$ persists upto $1.5<T / T_{c}<2.3$.

Thus, on the basis of lattice studies, the following picture emerges: The higher excited states dissociate around $T=T_{c}$, while the $J / \psi$ survives up to much higher temperature, in accord with the potential model studies based on the internal energy $U(r, T)$.

There is, however, a caveat to these calculations. The discretization introduced by the lattice limits the resolution of the peak. Lattice methods are thus useful in determining the position and to some extent the amplitude of the peaks, but determining the peak widths remains a challenge, nor is it easy to study the spectrum in the continuum region $(\omega>4 \mathrm{GeV})$. 


\subsection{Dynamics of quarkonium dissociation}

We have seen in the previous discussion that the $J / \psi$, the vector ground state of charmonium family, is very tightly bound. Its binding energy i.e. the energy difference between $J / \psi$ mass and open charm threshold, $\Delta E_{J / \psi}$, is considerably larger than the typical non-perturbative hadronic scale $\Lambda_{Q C D}$,

$$
\Delta E_{J / \psi}=2 M_{D}-M_{J / \psi}=0.6 \mathrm{GeV} \gg \Lambda_{\mathrm{QCD}} \sim 0.2 \mathrm{GeV}
$$

Consequently the size of $J / \psi$ is much smaller than that of typical hadron,

$$
r_{J / \psi} \sim 0.25 \mathrm{fm} \ll \Lambda_{\mathrm{QCD}}^{-1} \sim 1 \mathrm{fm}
$$

We now want to consider by what kind of dynamical interaction such a state can be dissociated. Because of the small spatial size, the $J / \psi$ can only be resolved by a sufficiently hard probe. Moreover, because of its high binding energy, only a sufficiently energetic projectile can break the binding. The previous study of global medium effects had led to the conclusion that only a hot deconfined medium, consisting of colored quarks and gluons, is capable of dissociating the charmonium vector ground state. We now want to study this on a microscopic level.

In a deconfined medium, the constituents are unbound partons, whereas in a confined medium the constituents are hadrons. Such thermal hadrons are incapable of causing collisional dissociation of $J / \psi$. Let us illustrate this point.

Consider the collision of a $J / \psi$ with a normal hadrorn. Because of the small characteristic $J / \psi$ size, only a hard partonic constituent of the hadron can see the $J / \psi$ and interact with it. In other words, $J / \psi$ collisions with ordinary hadrons probe the local partonic structure of these 'light' hadrons, not their global aspects such as mass, size, or overall momentum. The parton nature of the interaction is illustrated in Fig. 1.8

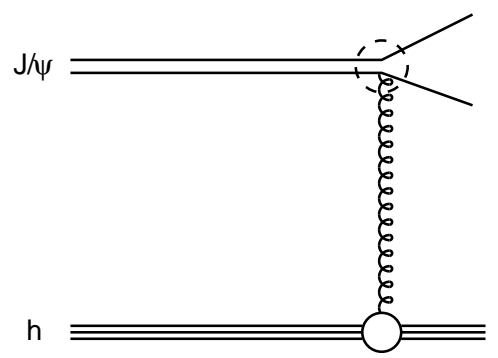

Fig. 1.8. Schematic view of the interaction of a normal hadron with a $J / \psi$.

To see the effect of this more quantitatively 17, we take an ideal pion gas as the confined medium. The momentum distribution of the pions at a temperature $T$ follows $f(p) \sim \exp (-|p| / T)$, giving the pions an average momentum $\langle p\rangle \sim 3 T$. Now the gluon momentum distribution inside a hadron as determined by deep inelastic lepton-hadron scattering is given by the parton distribution function $g(x)$; here $x=2 k_{g} / \sqrt{s}$, with $k_{g}$ for the gluon momentum, so that $x$ may be thought of as the 
fraction of the incident hadron's momentum carried by the gluon. For the pionic gluon it takes the form

$$
g(x) \sim(1-x)^{3}
$$

The resulting average gluon momentum in the hadron thus becomes

$$
\left\langle k_{g}\right\rangle=p_{h} \cdot \frac{\int_{0}^{1} d x x g(x)}{\int_{0}^{1} d x g(x)} .
$$

With eq. (1.10) and $p_{h}=3 T$ for the momentum of the incident hadron, we obtain

$$
\left\langle k_{g}\right\rangle_{h}=\frac{p_{h}}{5}=\frac{3 T}{5} \leq 0.1 \mathrm{GeV},
$$

where we have assumed $T<175 \mathrm{MeV}$ for the temperature of the hadronic medium. Thus gluons bound inside the hadronic constituents of confined matter are much too soft to cause the dissociation of a $J / \psi$.

On the other hand, in a deconfined medium, such as an ideal QGP, the gluons are free and distributed according to a thermal distribution $f\left(k_{g}\right) \sim \exp \left(-k_{g} / T\right)$, which gives

$$
\left\langle k_{g}\right\rangle \sim 3 T
$$

so that for $\mathrm{T} \geq 1.2 T_{c} \simeq 0.63 \mathrm{GeV}$, the gluons are hard enough to overcome the $J / \psi$ binding.

We have thus noticed that deconfinement results in a hardening of the relevant gluon momentum distributions. More generally speaking, the onset of deconfinement will lead to parton distribution functions which are different from those for free hadrons, as determined by DIS experiments. Since hard gluons are needed to resolve and dissociate $J / \psi$ 's , one can use $J / \psi$ s to probe the in-medium gluon hardness and hence the confinement status of the medium.

This qualitative picture can be made quantitative by short distance QCD calculations 17, 18. One has to calculate first the cross section for gluon dissociation of $J / \psi$, a QCD analogue of the photo-effect. This can be carried out using the operator product expansion, which is essentially a multipole expansion for the charmonium quark-antiquark system. Fig. 1.8 shows the relevant diagram for the calculation of inelastic $J / \psi$-hadron cross section. The upper part of the figure corresponds to $J / \psi$ dissociation by gluon interaction. The cross section for this process has the form

$$
\sigma_{g-J / \psi} \sim \frac{1}{m_{c}^{2}} \frac{\left(k / \Delta E_{\psi}-1\right)^{3 / 2}}{\left(k / \Delta E_{\psi}\right)^{5}}
$$

with $\Delta E_{J / \psi}=2 M_{D}-M_{J / \psi}$. The corresponding cross section for the hadron dissociation is obtained by convoluting this gluon dissociation cross section with the gluon distribution function $g(x)$ of the incident hadron. For $J / \psi$-meson interactions, this leads to the form

$$
\sigma_{h-J / \psi} \simeq \sigma_{\text {geom }}\left(1-\frac{\lambda_{0}}{\lambda}\right)^{5.5}
$$

with $\lambda \simeq\left(s-M_{\psi}^{2}\right) / M_{\psi}$ and $\lambda_{0} \simeq\left(M_{h}+\Delta E_{\psi}\right)$, where $\sqrt{s}$ is the CMS energy of the $J / \psi$-hadron system. Here $\sigma_{\text {geom }} \simeq \pi r_{J / \psi}^{2} \simeq 2 \mathrm{mb}$ is the geometric $J / \psi$ cross section and $M_{h}$ denotes the mass of the incident meson. Fig. 1.9 compares the two 


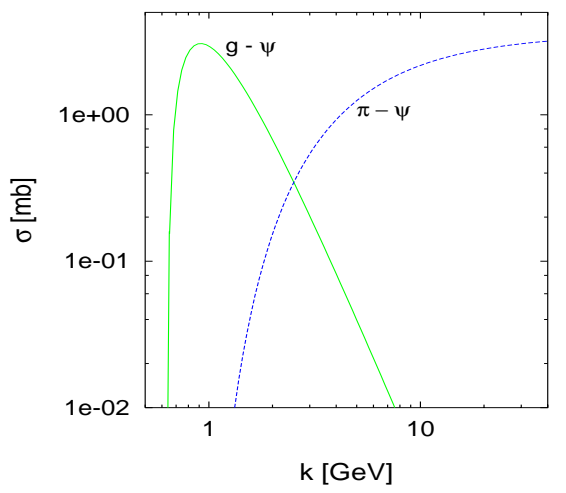

Fig. 1.9. Cross sections for $J / \psi$ dissociation by gluons vs. pions

\begin{abstract}
dissociation cross sections, $J / \psi$ dissociation by gluons (gluo-effect) and by pions, as a function of projectile momentum $k$ incident on stationary $J / \psi$, as given by eqs. (1.14) and (1.15). The gluon cross section shows the typical photo-effect form, vanishing until the gluon momentum $k_{g}$ reaches the binding energy $\Delta E_{J / \psi}$; it peaks just a little later $\left(\lambda_{g} \sim r_{J / \psi}\right)$ and then vanishes again when sufficiently hard gluons just pass through the (comparatively larger) charmonium bound states $\left(\lambda_{g} \ll r_{J / \psi}\right)$. In contrast, the $J / \psi$-hadron inelastic cross section remains negligibly small until rather high hadron momenta (3-4 GeV). In a thermal medium such momenta corresponds to temperatures of more than $1 \mathrm{GeV}$. In other words, in a confined medium in the temperature range of the order of a few hundred $\mathrm{MeV}$ the $J / \psi$ should survive, but it should become dissociated in a hot deconfined medium. Confined media in the temperature range of few hundred $\mathrm{MeV}$ are thus essentially transparent to a $J / \psi$, while a deconfined medium of the same temperature is opaque to $J / \psi$ 's and very efficiently dissociates them.
\end{abstract}

\title{
1.4 Quarkonium production in nuclear collisions
}

The aim of ultra-relativistic nuclear collisions is to study color deconfinement and the resulting quark-gluon plasma in the laboratory. We want to use quarkonia produced in the collision as a probe to study the medium produced in the collision. Both the quarkonium states and the medium to be probed require a 'finite formation time', so we have to look at the evolution aspects in both cases. Let us first consider the issue of charmonium production in hadron-hadron collisions and then turn to nuclear targets.

\subsubsection{Quarkonium production in hadronic collisions}

Quarkonium production in hadron-hadron collisions occurs in three stages. The first stage is the production of $c \bar{c}$ pair. Because of the large quark mass $\left(m_{c} \sim 1.3 \mathrm{GeV}\right)$ this process can be treated as a hard process and is well described by perturbative 
QCD. A parton from the projectile interacts with one from the target; the (nonperturbative) parton distributions within the hadrons are determined empirically in other reactions, e.g., by deep inelastic lepton-hadron scattering. At high energy the process of $c \bar{c}$ production dominantly occurs by gluon fusion, $g g \rightarrow c \bar{c}$ (see Fig. 1.10).

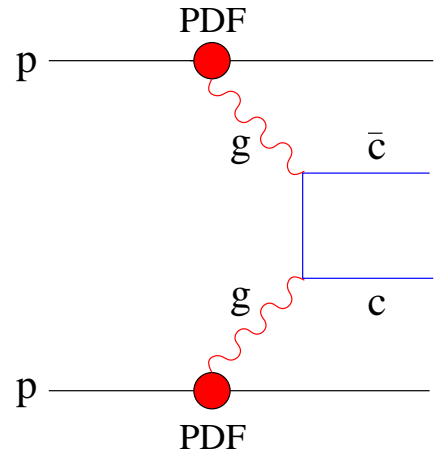

Fig. 1.10. Lowest-order Feynman diagram for $c \bar{c}$ production through gluon fusion.

The $c \bar{c}$ in general is in a color octet state. It has to neutralize its color in order to leave the interaction zone and form a physical resonance like $J / \psi$ or $\psi^{\prime}$. In the second stage, color neutralization occurs by interaction with the surrounding color field. This results finally in the third stage of a physical bound state. Both the second and third stages are non-perturbative in nature.

On a fundamental theoretical level, color neutralization is not yet fully understood, but there are several models, color singlet [19, color octet [20, and color evaporation [21]. The color evaporation model provides a particularly simple and experimentally well-supported phenomenological approach. In the evaporation process, the $c \bar{c}$ can either combine with light quarks to form open charm mesons $(D$ and $\bar{D}$ ) or bind with each other to form a hidden charm (charmonium) state. A fixed fraction of the subthreshold $c \bar{c}$ production is used in charmonium production. The basic quantity in this picture is the total sub-threshold charm cross section $S_{c \bar{c}}$, obtained by integrating the perturbative $c \bar{c}$ production cross section $\sigma$ over the mass window from $2 m_{c}$ to $2 m_{D}$. Since at high energy, the dominant part of $S_{c \bar{c}}$ comes from gluon fusion (Fig. 1.10), we can write

$$
S_{c \bar{c}}(s) \simeq \int_{2 m_{c}}^{2 m_{D}} d \hat{s} \int d x_{1} d x_{2} g_{p}\left(x_{1}\right) g_{t}\left(x_{2}\right) \sigma(\hat{s}) \delta\left(\hat{s}-x_{1} x_{2} s\right),
$$

with $g_{p}(x)$ and $g_{t}(x)$ denoting the gluon densities and $x_{1}$ and $x_{2}$ the fractional momenta of the gluons from projectile and target, respectively; $\sigma$ is the $g g \rightarrow c \bar{c}$ cross section.

As mentioned, the basic assumption of the color evaporation model is that the production cross section for any particular charmonium state is a fixed fraction of the subthreshold charm cross section, 


$$
\sigma_{i}(s)=f_{i} S_{c \bar{c}}(s)
$$

where $f_{i}$ is an energy-independent constant to be determined empirically. It follows that the energy dependence of the production cross section for any charmonium state is predicted to be that of the perturbatively calculated sub-threshold charm cross section. As a further consequence the production ratios of different charmonium states

$$
\frac{\sigma_{i}(s)}{\sigma_{j}(s)}=\frac{f_{i}}{f_{j}}=\text { constant }
$$

must be energy independent. Both these predictions have been compared in detail to charmonium hadro-production data over a wide range of energies 22 . They are found to be well supported, both in the energy dependence of the cross sections and in the constancy of the relative species abundances.

Before turning to the topic of quarkonium production in hadron-nucleus collisions, let us consider the relevant time scales for the $J / \psi$ formation.

The formation of a $c \bar{c}$ pair requires a time $\tau_{c \bar{c}}=1 / 2 m_{c}=0.05 \mathrm{fm}$. The produced $c \bar{c}$ pair is in a color-octet state. To form a physical resonance state, it has to neutralize its color. The color-octet model [20] proposes that the color-octet $c \bar{c}$ combines with a soft collinear gluon to from a color-singlet $(c \bar{c}-g)$ state. After a short relaxation time $\tau_{8}$ this pre-resonance $(c \bar{c}-g)$ turns into physical resonance by absorbing the accompanying gluon, with similar formation processes for the other resonances, such as $\chi_{c}$ and $\psi^{\prime}$ production. The color-octet model encounters difficulties if the collinear gluons are treated perturbatively, indicating once more that color neutralization seems to require non-perturbative elements. However it does provide a conceptual basis for the evolution of the formation process.

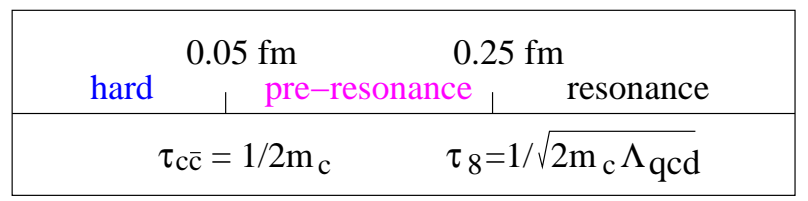

Fig. 1.11. Evolution of $J / \psi$ formation in a hadron-hadron collision

The color neutralization time $\tau_{8}$ of the pre-resonant state can be estimated by the lowest momentum possible for the confined gluons $\tau_{8} \simeq\left(2 m_{c} \Lambda_{Q C D}\right)^{-1 / 2} \simeq 0.25$ $\mathrm{fm}$. The resulting scales of $J / \psi$ formation are illustrated in Fig(1.11). The formation time for the actual physical ground state $J / \psi$ is presumably somewhat larger than $\tau_{8}$, although $r_{J / \psi} \simeq \tau_{8}$, the heavy $c$ quarks move non-relativistically. For the larger higher excited states, the formation time will then be correspondingly still larger.

\subsubsection{Quarkonium production in $\mathrm{pA}$ and AA collisions}

Let us now turn to nuclear collisions. Both in p-A and A-A interactions there will be pre-resonant absorption in nuclear matter. In nucleus-nucleus collisions, however, 
there can be in addition a substantial amount of a produced "secondary medium", and testing this medium is in fact our main objective.

The creation of the medium and production of the probe lead to two distinct formation scales. In p-A collision there is no formation time for the medium, so that such collisions provide a tool to probe charmonium production, evolution and absorption in confined matter.

Nuclear effects can arise in all the evolution stages of $J / \psi$ production, and a number of different phenomena have to be taken into account.

- The presence of other nucleons in the nucleus can modify the initial state parton distribution functions, which enter in the perturbative $c \bar{c}$ production process, as shown in Fig. 1.10 This can lead to a decrease (shadowing) or to an increase (antishadowing) of the production rate.

- Once it is produced, the $c \bar{c}$ pair in its evolution will traverse the nuclear matter; it can suffer absorption both in the pre-resonance as well as in the resonance stage, caused by successive interactions with the target nucleons.

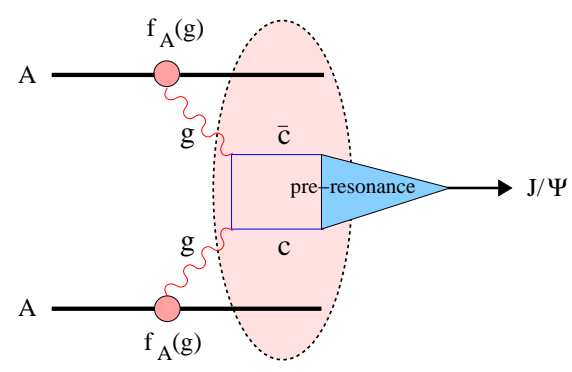

Fig. 1.12. $J / \psi$ production in a nuclear medium.

Hence $J / \psi$ production in a nuclear medium is modified as compared to hadronic collisions. The modification occurs before any QGP formation and is thus independent of the effects due to a deconfined medium having free quarks and gluons. If we want to use $J / \psi$ production and its suppression in a nuclear collision as a potential signature of the QGP formation, all normal nuclear effects must first be taken into account. Only then can charmonium suppression serve as a probe to test the confining status of the produced "secondary medium" in nuclear collisions.

So an essential question is how to account for the effects of the nuclear medium initially present on the production. The basis for this, both in theory and in experiment, is the measurement of dilepton, open charm and charmonium production in $\mathrm{p}-\mathrm{A}$ or $\mathrm{d}-\mathrm{A}$ collisions. These collisions thus provide a crucial tool to understand quarkonium production in nuclear collisions.

The procedure to be used for such studies is the following:

- The initial state parton distribution functions in nuclear matter are determined by open charm and dilepton production in $\mathrm{p}-\mathrm{A} / \mathrm{d}-\mathrm{A}$ collisions in the relevant kinematic region. 
- The Glauber model then is used to determine the pre-resonance absorption of the $J / \psi$ and $\psi^{\prime}$ by the target nucleon in $\mathrm{p}-\mathrm{A} / \mathrm{d}-\mathrm{A}$ in the relevant kinematic region.

It is thus clear that $\mathrm{p}-\mathrm{A}$ or $\mathrm{d}-\mathrm{A}$ collision experiments are an absolutely essential tool for the analyis of quarkonium production in nuclear collisions.

\subsubsection{Sequential quarkonium suppression}

There is a further important and, as it turns out, crucial feature observed in $J / \psi$ hadroproduction. The $J / \psi$ actually measured in hadron-hadron collisions are not all directly produced $1 S$ charmonium states; rather, they have three distinct origins. About $60 \%$ of them are indeed directly produced $1 \mathrm{~S}$ charmonium states, but the rest are feed-down from higher excited states. About $30 \%$ come from the decay $\chi_{c}(1 P) \rightarrow J / \psi+$ anything and the remaining $10 \%$ from $\psi^{\prime}(2 S) \rightarrow J / \psi+$ anything. In both cases, the decay widths of the involved higher excited states are extremely small (less than $1 \mathrm{MeV}$ ), so that their lifetimes are very long and the decay occurs long after the interaction. The presence of any medium in nuclear collisions would therefore affect these excited states themselves and not their products, and we had seen above that excited states are dissociated before the ground state is. This has a direct consequence on the nature of $J / \psi$ suppression by deconfinement. In a thermal QCD medium, we should expect that with increasing temperature or energy density, first the $J / \psi$ originating from $\psi^{\prime}$ decay and then those from $\chi_{c}$ decay will disappear. Only a considerably higher temperature would be able to remove the directly produced $J / \psi$ s. Such a stepwise onset of suppression with specified threshold temperatures is perhaps the most characteristic feature predicted for charmonium production in nuclear collisions. It is illustrated schematically in Fig. 1.13, where we have defined the $J / \psi$ survival probability to be unity if the production rate suffers only the estimated normal nuclear suppression. The generic suppression pattern shown here will of course be softened by nuclear profile effects, impact parameter uncertainties, etc. On the other hand, this could be partially compensated if there is a discontinuous onset of deconfinement as a function of energy density of the medium.

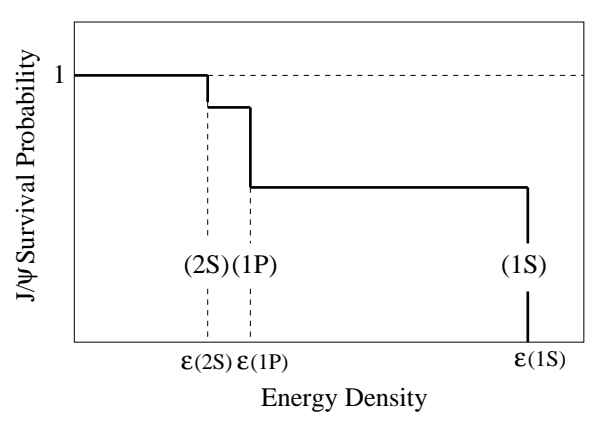

Fig. 1.13. Sequential $J / \psi$ suppression 
We had seen above how to calculate the quarkonium dissociation points which specify the temperature and thus also the energy density of the medium, thereby serving as a QGP thermometer. Potential model studies based on the heavy quark internal energy, as well as direct lattice QCD calculations gave as dissociation temperatures $T \simeq 1.1 T_{c}$ for $\psi^{\prime}$ and $\chi_{\mathrm{c}}$ and $T \geq 1.5-2 T_{c}$ for $\mathrm{J} / \psi$. If this is correct, then the direct $J / \psi(1 \mathrm{~S})$ survives up to about $\epsilon \geq 10-20 \mathrm{GeVfm}^{3}$. Consequently, all anomalous suppression observed at SPS and RHIC must be due to the dissociation of higher excited states $\chi_{c}$ and $\psi^{\prime}$ [23]. The suppression onset for this is predicted to lie around $\epsilon \simeq 1 \mathrm{GeV} / \mathrm{fm}^{3}$, and once these are gone, only the unaffected $J / \psi$ (1S) production remains. Hence the $J / \psi$ survival probability (once normal nuclear effects are taken into account) should be same for central $\mathrm{Au}-\mathrm{Au}$ at $\mathrm{RHIC}$ as for central $\mathrm{Pb}-\mathrm{Pb}$ collisions at SPS.

\subsubsection{Charmonium regeneration}

In this section we want to investigate the possibility that the medium produced in high energy nuclear collision differs from the deconfined state of matter studied in finite temperature QCD. The basic idea here is that nuclear collisions initially produce more than the thermally expected charm and this excess, if it survives, may lead to a new form of combinatorial charmonium production at hadronization [24, 25, 26].

In the QGP argumentation, a crucial aspect was that the charmonia, once dissociated, can not be recreated at the hadronization stage, because of the extremely low thermal abundance of charm quarks in an equilibrium QGP. The thermal production rate for a $c \bar{c}$ pair relative to a pair of light quarks is

$$
\begin{aligned}
\frac{n_{c \bar{c}}}{n_{q \bar{q}}} & \simeq \exp -\left(2 m_{c}-2 m_{q} / T_{c}\right) \\
& \simeq \exp \left(-2 m_{c} / T_{c}\right) \simeq 3.5 \times 10^{-7},
\end{aligned}
$$

with $m_{c}=1.3 \mathrm{GeV}$ for charm quark mass and $T_{c}=175 \mathrm{MeV}$ for the transition temperature. The initial charm production in high energy hadronic collisions is, however, a hard non-thermal process, and the resulting rates calculated from perturbative QCD are considerably larger than the thermal rate. Moreover, in AA interactions the resulting $c / \bar{c}$ production rate grows with the number of binary collision $N_{\text {coll }}$, while the light quark production rate, being soft process, grow as the number of participants nucleons, i.e much slower. At high collision energies, the initial charm abundance in AA collisions is thus very much higher than the thermal value. Now the question is, what happens to this in course of the collision evolution?

The basic assumption of the regeneration approach is that the initial charm excess is maintained throughout subsequent evolution, i.e, the initial chemical nonequilibrium will persist up to the hadronization point. In charmonium hadroproduction, $J / \psi$ are formed because some of the $c \bar{c}$ pairs produced in a given collision form the corresponding bound state. In a collective medium formed through the superposition of many nucleon-nucleon (NN) collisions, such as a quark-gluon plasma, a $c$ quark from one NN collision can in principle also bind with a $\bar{c}$ from another NN collision ("new" pairs) to create a $J / \psi$. This pairing provides a "exogamous" charmonium production mechanism, in which the $\mathrm{c}$ and $\bar{c}$ in a charmonium state have different parents, in contrast to "endogamous" production in pp collision. At 
sufficiently high energies this can lead to an enhancement in $J / \psi$ production in AA collisions compared to a scaled pp rates [24, 25, 26], provided the overall charm density is sufficiently high at hadronization and provided the binding probability between charm quarks from different sources is large enough.

Whether or not such enhancement becomes significant depends on two factors. On one hand, the initial charm oversaturation must be preserved so that the total charm abundance is non-thermal. On the other hand it is necessary that the recombination between charm quarks from different parents to charmonium $(J / \psi)$ is strong enough. Here it is generally assumed that the final hadronization occurs according to the available phase space. Thus the number of statistically recombined $J / \psi$ has the form $N_{J / \psi} \sim N_{c \bar{c}}^{2}$, growing quadratically in the number of $c \bar{c}$ pairs. This implies that the hidden to open charm ratio, e.g., $N_{J / \psi} / N_{D} \sim N_{c \bar{c}} / N_{h}$ increases with energy, in contrast to the energy independent form obtained for the fully equilibrated QGP, or to the decrease predicted by color screening. The prediction for $J / \psi$ production by regeneration is compared in Fig. 1.4.4 to that from sequential suppression.

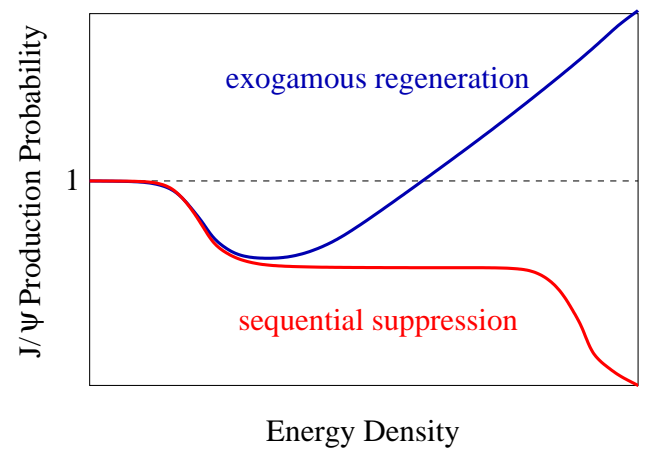

Fig. 1.14. Statistical $J / \psi$ regeneration vs. sequential $J / \psi$ suppression

\subsection{Conclusion}

Statistical QCD predicts the existence of a new state of nuclear matter, the quarkgluon plasma (QGP), at very high temperatures and/or densities. This medium, in contrast to hadronic matter, is capable of dissociating quarkonia, so that quarkonium suppression may be taken as a sign of QGP formation in nuclear collisions 3 . Furthermore, different quarkonia dissociate at different temperatures; the dissociation pattern thus serves as a "thermometer" for the QGP. It is therefore important to obtain precise predictions for these dissociation points, and for this, one can turn to either of two approaches, potential models or lattice studies. The former have the problem that the results are dependent on the type of potential chosen, while the latter so far suffer from the fact that lattice spacing and statistics limits the resolution of peak widths in the spectrum. It is also not easy to identify the continuum region of the spectrum on the lattice. 
But what happens in case of relativistic nuclear collisions in the laboratory? If there is no regeneration of the dissociated charmonia, $J / \psi$ remain as an external probe, and the sequential suppression pattern of the $J / \psi$ can then serve as a tool to determine the energy density and the temperature of the produced medium. On the other hand, if there is $J / \psi$ production through statistical combination of $c$ and $\bar{c}$ from different collisions, leading to an overall $J / \psi$ enhancement, this would clearly indicate the thermalization of the produced medium on a pre-hadronic level. However, charmonia could then no longer serve as a thermometer to charaterize the primordial medium. Data from LHC, soon to come, will certainly play a decisive role in settling the issue.

\section{References}

1. E. Eichten et al., Phys. Rev. D17 (1978) 3090; Phys. Rev. D 21 (1980) 203.

2. For a recent review, see H. Satz, J. Phys. G 32 (2006) R25.

3. T. Matsui and H. Satz, Phys. Lett. B, 178, 416 (1986).

4. For a recent review, see H. Satz, Nucl. Phys. A 783 (2007) 249c.

5. F. Karsch, M.-T. Mehr and H. Satz, Z. Phys. C 37 (1988)

6. S. Digal, P. Petreczky and H. Satz, Phys. Lett. B 514 (2001) 57.

7. E. Shuryak and I. Zahed, Phys. Rev. D 70, 054507 (2004).

8. C.-Y. Wong, Phys. Rev. C 72 (2004) 034906; C.-Y. Wong, hep-ph/0509088.

C.-Y. Wong, Phys. Rev. C 76 (2007) 014902.

9. W. Alberico et al., Phys. Rev. D 72 (2005) 114011.

10. S. Digal et al., Europ. Phys. J. C 43 (2005) 71.

11. T. Umeda et al., Int. J. Mod. Phys. A16 (2001) 2215.

12. M. Asakawa and T. Hatsuda, Phys. Rev. Lett. 92 (2004).

13. S. Datta et al., Phys. Rev. D 69 (2004) 094507

14. H. Iida et al., PoS LAT2005 (2006) 184.

15. A. Jacovac et al., Phys. Rev. D 75 (2007) 014506.

16. R. Morrin et al., PoS LAT2005 (2006) 176;

G. Aarts et al., Nucl. Phys. A 785 (2007) 198.

17. D. Kharzeev and H. Satz, Phys. Lett. ¡b334 (1994) 155.

18. M. E. Peskin, Nucl. Phys. B 156 (1979) 365;

G. Bhanot and M. E. Peskin, Nucl. Phys. B 156 (1979) 391.

19. R. Baier and R. Rückl, Z. Phys. C 19 (1983) 251.

20. G. T. Bodwin, E. Braaten and G. P. Lepage, Phys. Rev. D 51 (1995) 1125;

E. Braaten and S. Fleming, Phys. Rev. Lett. 74 (1995) 3327.

21. M. B. Einhorn and S. D. Ellis, Phys. Rev. D12 (1975) 2007;

H. Fritzsch, Phys. Lett. 67B (1977) 217;

M. Glück, J. F. Owens and E. Reya, Phys. Rev. D17 (1978) 2324;

J. Babcock, D. Sivers and S. Wolfram, Phys. Rev. D18 (1978) 162.

22. H. Satz and X.-N. Wang (Eds.), Hard Processes in Hadronic Interactions, Int. J. Mod. Phys. A 10 (1995) 2881;

M. Mangano et al. (Eds.), Hard Probes in Heavy-Ion Collisions at the LHC, CERN Yellow Report 2004-09, Geneva 2004.

23. D. Kharzeev, F. Karsch and H. Satz, Phys. Lett. B 637 (2006) 75.

24. P. Braun-Munzinger and J. Stachel, Nucl. Phys. A690 (2001) 119.

25. R. L. Thews et al., Phys. Rev. C 63 (2001) 054905.

26. L. Grandchamp and R. Rapp, Nucl. Phys. A 709 (2002) 415. 